\title{
Resonances in a circular dielectric cavity
}

\author{
Jung-Wan Ryu ${ }^{1} *$ Sunghwan Rim², Young-Jai Park ${ }^{2}$, Chil-Min Kim², and Soo-Young Lee H $^{2}$ \\ 1 Department of physics, Pusan National University, Busan, 609-735, Korea \\ 2 Department of Physics, Sogang University, Seoul 121-742, Korea and \\ 3 School of Physics and Astronomy, \\ Seoul National University, Seoul, 151-742, Korea
}

\begin{abstract}
We study resonance distributions in a circular dielectric cavity. It is shown that the decay-rate distribution has a peak structure and the details of the peak are consistent with the classical survival probability time distribution. We also investigate the behavior of the complex resonance positions at the small opening limit ( $n \rightarrow \infty, n$ is the refractive index of the cavity). At the large $n$ limit, the real part of complex resonance positions approaches the solutions with different $m$ of Dirichlet problem with a scale $n^{-2}$ and the imaginary part goes zero as $n^{-2 m}$ for TM and $n^{-2(m+1)}$ for TE polarization, where $m$ is the order of the resonance.

PACS numbers: 42.55.Sa, 42.60.Da, 05.45.Mt
\end{abstract}

${ }^{*}$ E-mail address:jungwanryu@pnu.ac.kr

${ }^{\dagger}$ Corresponding author. Tel:+82-2-880-1468, Fax: +82-2-876-3973, E-mail address: pmzsyl@naver.com 
Two-dimensional (2-D) billiards with various boundary geometries have been widely studied in quantum chaos community because of the ease in analysis and rich interesting phenomena such as classical and wave chaos and wave localization etc. There are many relevant experimental realizations, e.g., surface waves [1], microwave billiards [2, 3], mesoscopic structures [4, 5, 6], and semiconductor microcavities [7, 8, 9, 10, 11]. In experiments, the systems measured are inevitably coupled with environment, i.e., they are open. Even in the case of a small coupling, it is sometimes difficult to explain the open system based on the physical properties of the corresponding closed system without a clear understanding of the correspondence between them.

The decay-rate statistics is determined by interpaly of openness and dynamics of the system concerned[12]. In practical purpose, it is, however, not a simple task to obtain many decay rates enough to apply statistical processes for a chaotic open quantum system, such as a chaotic dielectric cavity, because of heavy numerical tasks. From the viewpoint of a easy analysis, the circular dielectric cavity is a good open system, due to its simple geometry, to study the statistics of decay rates which originate from the dielectric property and the relationship to the corresponding closed billiard with the Dirichlet boundary condition. In dielectric cavities the degree of opening is determined by the refractive index $n$ and the small opening limit corresponds to $n \rightarrow \infty$.

In this letter, we answer the following two questions about resonances in a circular dielectric cavity: (i) How are the imaginary values of resonance positions, which represent the decay rates of the resonances, in the circular dielectric cavity distributed? (ii) Would its resonance positions approach the eigenvalues of the corresponding billiard at the small opening limit? For the first question, we obtain the complex resonance positions and show that the distribution of imaginary values is consistent with survival probability time distribution (SPTD) which represents the decay property due to classical ray escapes [13]. As for the second question, we show that the resonance of TM polarization with angular quantum number $m$ in circular dielectric cavity approaches the eigenvalues with $m-1$, not $m$, of Dirichlet problem at the small opening limit $(n \rightarrow \infty)$.

From the mathematical viewpoint, the only difference between closed billiard and open dielectric cavity problems is the boundary condition applied to the Helmholtz equation [14],

$$
\left(\nabla^{2}+k^{2}\right) \psi=0
$$


where $k$ is the wavenumber inside the billiard or the dielectric cavity. In the closed billiard problem, the typical boundary conditions are Dirichlet and Neumann boundary conditions,

$$
\begin{aligned}
& \text { (Dirichlet) } \psi\left(\mathbf{r}_{b}\right)=0, \\
& \text { (Neumann) } \partial_{n} \psi\left(\mathbf{r}_{b}\right)=0
\end{aligned}
$$

where $\mathbf{r}_{b}$ denotes the boundary vector and $\partial_{n}$ is the normal derivative to the boundary. For an arbitrary boundary shape we can find real eigenvalues $k_{n}$ and the eigenfunctions $\psi_{n}(\mathbf{r})$ satisfying the corresponding boundary condition. In the dielectric cavity case, we have to apply different boundary conditions depending on the polarization. For TM (TE) polarization, the electric (magnetic) field normal to the 2-D cavity and its normal derivative (its normal derivative divided $n^{2}$ ) are continuous at the boundary interface, i.e.,

$$
\begin{aligned}
& \text { (TM) } \frac{\psi_{\text {in }}\left(\mathbf{r}_{b}\right)}{\partial_{n} \psi_{\text {in }}\left(\mathbf{r}_{b}\right)}=\frac{\psi_{\text {out }}\left(\mathbf{r}_{b}\right)}{\partial_{n} \psi_{\text {out }}\left(\mathbf{r}_{b}\right)} \\
& \text { (TE) } \frac{n_{\text {in }}^{2} \psi_{\text {in }}\left(\mathbf{r}_{b}\right)}{\partial_{n} \psi_{\text {in }}\left(\mathbf{r}_{b}\right)}=\frac{n_{\text {out }}^{2} \psi_{\text {out }}\left(\mathbf{r}_{b}\right)}{\partial_{n} \psi_{\text {out }}\left(\mathbf{r}_{b}\right)}
\end{aligned}
$$

where $\psi_{\text {in }}(\mathbf{r})$ and $\psi_{\text {out }}(\mathbf{r})$ are wave functions inside and outside the dielectric cavity, respectively, and the refractive indices of the cavity and environment are $n_{\text {in }}$ and $n_{\text {out }}$. We will set $n_{\text {in }}=n$ and $n_{\text {out }}=1$ throughout the letter. The resonance positions $k$, obtained from the above boundary conditions, are complex numbers with negative imaginary values. The quality $(\mathrm{Q})$ factor of the corresponding resonance mode is defined as $-\operatorname{Re}(k) / 2 \operatorname{Im}(k)$. High Q resonance modes, therefore, have smaller absolute values of $\operatorname{Im}(k)$ and mean the resonance modes well confined inside the cavity.

For the circular boundary geometry with a radius $R$, the Helmholtz equation, Eq.(1), can separate into the angular and radial equations due to the rotational symmetry, and the solution of the angular equation is simply given by $e^{i m \phi}$ ( $m$ is an integer). The radial equation is represented by Bessel differential equation [15], and relevant solution inside both the circular billiard and dielectric cavity is Bessel function, $J_{m}(k r)$. In the circular dielectric cavity, the solution outside the cavity should be outgoing wave which is represented by Hankel function of the first kind, $H_{m}^{(1)}(k r)$. Using these solutions, the boundary conditions (Eq.(2) , Eq.(3) ) become [14, 16, 17]

$$
\begin{aligned}
& \text { (Dirichlet) } J_{m}(k)=0, \\
& (\text { Neumann }) k J_{m-1}(k)-m J_{m}(k)=0,
\end{aligned}
$$




$$
\begin{aligned}
& n J_{m-1}(k) H_{m}^{(1)}\left(k_{0}\right)-J_{m}(k) H_{m-1}^{(1)}\left(k_{0}\right)=0, \\
& n J_{m}(k) H_{m-1}^{(1)}\left(k_{0}\right)-J_{m-1}(k) H_{m}^{(1)}\left(k_{0}\right) \\
& =\frac{m}{k_{0}}\left(n-\frac{1}{n}\right) J_{m}(k) H_{m}^{(1)}\left(k_{0}\right),
\end{aligned}
$$

where we take $R=1$ without loss of generality, and $k_{0}$ is the vacuum wavenumber and $k_{0}=k / n$. In general, the separability of the Helmholtz equation implies that the circular system is integrable and there are good quantum numbers specifying the solution. In our case, the solution can be classified by the angular quantum number $m$ and the radial quantum number $l$ as $k_{(m, l)}$. Figure 1 shows eigenfunctions satisfying Dirichlet (a) and Neumann (b) boundary conditions and resonance modes in TM (c) and TE (d) cases, and these are specified by a mode index $( \pm m, l)=( \pm 8,1)$. The eigenvalues and the resonance positions are $k_{D( \pm 8,1)}=12.2251(\mathrm{a}), k_{N( \pm 8,1)}=9.6474(\mathrm{~b}), k_{T M( \pm 8,1)}=10.7845-i 0.02278(\mathrm{c})$, and $k_{T E( \pm 8,1)}=11.6295-i 0.03993$, where we take $n=2$ in TM and TE cases. It is easily seen that the numbers of high intensity spots along the perimeter (angular direction) and along the radial direction are $2 m$ and $l$, respectively. As expected from the above example, the eigenvalues and the resonance positions for one mode index $( \pm m, l)$ have an order as $k_{D( \pm m, l)}>\operatorname{Re}\left[k_{T E( \pm m, l)}\right]>\operatorname{Re}\left[k_{T M( \pm m, l)}\right]>k_{N( \pm m, l)}$.

We confirm numerically that all resonances in the TM case can be classified by the mode index $( \pm m, l)$ just like the Dirichlet and Neumann cases. However, we find that in the TE case there are additional modes with a nonzero angular momentum $m$, absent in other cases, which can not be classified by the mode index $( \pm m, l)$. Figure 2 shows an example of the additional modes, $( \pm m, l)=( \pm 8, \times)$ and $k_{T E( \pm 8, \times)}=17.3507-i 2.4802$. As expected from the figure and large absolute value of $\operatorname{Im}(k)$, these modes are very leaky and originated from the existence of the Brewster angle in TE case on which rays can transmit without reflection. Therefore, these modes do not show any notable wave confinement by the dielectric interface.

In order to investigate the distribution of resonance positions of the circular dielectric cavity, we obtain all solutions in the range of $0<\operatorname{Re}(k R)<150$ with $R=1$ and $n=2$ for both TM and TE cases. With the real part of the complex solutions obtained, we can check the level spacing distribution that is known to be Poisson and Wigner distribution for the integrable and chaotic billiards, respectively [6]. Although the circular dielectric cavity is an open system, it is still integrable, equivalent with the fact that all resonances can be specified by the mode index $(m, l)$. We can, therefore, expect that the level spacing distribution is 
Poissonic, and as shown in Fig. 3 the numerical calculation confirms this expectation.

The properties of openness in the circular dielectric cavity have been investigated by calculating the SPTD [13] which shows very different short time behaviors depending on the polarization. Especially, the exponential short time behavior appears in TE case and it has some relation to the existence of the Brewster angle. It is natural to relate this ray dynamical result with the distribution of imaginary values of the resonance positions since the imaginary value is relevant to the wave confinement by the dielectric cavity.

The distributions of imaginary values of resonance positions for both TM and TE cases are shown in Fig. 4 (a) and (b), respectively. The substantial difference between both cases is that the distribution for the TM case is bounded, i.e., has a minimum imaginary value $-\gamma_{M}(n) / 2$ while the result for the TE case is unbounded [18]. This result can be easily understood from details of reflection coefficients $R_{T M}(n, \theta)$ and $R_{T E}(n, \theta), \theta$ is the incident angle, determined by the Fresnel equations [19]. Consider an initial ray with a fixed incident angle $\theta$ which is invariant in the circular boundary. The survival probability of the ray would decay as $I(t)=e^{-\gamma(n, \theta) t}$ where the decay rate is given by

$$
\gamma(n, \theta)=-\frac{\ln R_{T M(T E)}(n, \theta)}{2 \cos \theta}
$$

and the time $t$ is scaled as the length of the ray trajectory. On the other hand, the intensity of a resonance decays as

$$
I(t)=\left|e^{-i \omega t}\right|^{2}=\left|e^{-i(\operatorname{Re}(k)+i \operatorname{Im}(k)) t}\right|^{2}=e^{2 \operatorname{Im}(k) t} .
$$

Therefore, the decay rate of a resonance mode $(m, l)$ is given by

$$
\gamma_{(m, l)}=-2 \operatorname{Im}\left(k_{(m, l)}\right)
$$

The ray dynamical (Eq.(5)) and resonance mode (Eq.(7)) decay rates have the same physical meaning due to the invariance of incident angle $\theta$ in the circular boundary case. Therefore, if $\gamma(n, \theta)$ in Eq.(5) is bounded, we can say that $\operatorname{Im}\left(k_{(m, l)}\right)$ is also bounded in TM case. We note that $R_{T M}(n, \theta)$ has its minimum at $\theta=0$, i.e., the case of normal incident rays or bouncing ball trajectories. Then, the minimum $\operatorname{Im}\left(k_{T M}\right)$ is $-\gamma_{M}(n) / 2$ and

$$
\gamma_{M}(n)=-\frac{\ln R_{T M}(n, \theta=0)}{2}=\ln \frac{n+1}{n-1} .
$$

The minimum imaginary value for the $n=2$ case is $-(\ln 3) / 2 \simeq-0.55$ which is consistent with Fig.4 (a). The above expression for the minimum imaginary value shows very good 
agreement with numerical results for various refractive indices, $n=2,4,6,8,10$, as shown in Fig 5 (a). On the other hand, in TE case there is the Brewster angle $\theta_{B}=\arctan (1 / n)$, and the ray incident with $\theta_{B}$ transmits without reflection, i.e., $R_{T E}\left(n, \theta_{B}\right)=0$. Then the decay rate $\gamma(n, \theta)$ in Eq.(5) becomes infinity at $\theta=\theta_{B}$, and the imaginary value of resonance position in TE case can have very large absolute value of $\operatorname{Im}(k)$ like the additional mode $( \pm m, \times)$ mentioned before. This fact is consistent with the numerical result in Fig. 4 (b).

We emphasize that the distributions of imaginary values of resonance positions, excepting the very high-Q resonances with only tunneling decay, can explain the behavior of the SPTD [13]. The resonance modes near the peak in Fig. 4 contribute to the short-time exponential decay in the SPTD, and the high-Q modes distributed just below the tunneling regime near zero in Fig. 4 do to the long-time algebraic behavior in the SPTD. In TM case, the fact that two mode-classes are smoothly connected, is consistent with the gradual transition from exponential to algebraic decays. On the other hand, in TE case, the distribution show an abrupt change at the peak, i.e., the two mode-classes are almost isolated, and this is responsible to the clear transition point from exponential to algebraic decays in SPTD.

In order to understand whole shape of the distributions, we plot the resonance positions $k_{(m, l)}$ with $m=40$ in Fig. 5 (b) [17, 18]. Black rectangle and red diamond represent resonance positions of TM and TE cases, respectively. It is known that the incident angle $\theta$ of waves in a resonance mode $(m, l)$ can be estimated by a semiclassical relation [7]

$$
\sin \theta=m / \operatorname{Re}\left(k_{(m, l)}\right)
$$

From this relation we can obtain some $\operatorname{Re}(k)$ values corresponding to the critical angle $\theta_{c}$ for total internal reflection and the Brewster angle $\theta_{B}$, which are indicated by black and red arrows, respectively, in Fig. 5 (b). If the incident angles of resonance modes calculated from Eq. (9) are greater than the critical angle $\theta_{c}$, waves inside are very well confined by total internal reflection and then the resonance mode has very small $|\operatorname{Im}(k)|$ as shown on the left of the black arrow. These high Q modes explain the peak near $|\operatorname{Im}(k)|=0$ in Fig. 4 (a) and (b), and the fraction of these high $\mathrm{Q}$ modes would be $(1-1 / n)$ in the semiclassical limit. As $\operatorname{Re}(k)$ increases beyond the black arrow, $\operatorname{Im}(k)$ converges gradually to the minimum value $-\gamma_{M} / 2$ in the TM case, which corresponds to the bounded distribution near the minimum value in Fig. 4 (a). In the TE case, near the red arrow corresponding to $\theta_{B}, \operatorname{Im}(k)$ shows very low value and then converges to $-\gamma_{M} / 2$, which explains the unbounded distribution 
and the peak at $-\gamma_{M} / 2$ in Fig. 4 (b). The same limit value $-\gamma_{M} / 2$ can be expected from the fact that $R_{T M}(n, \theta=0)=R_{T E}(n, \theta=0)$. The green and blue lines are obtained by calculating the relation between $\operatorname{Re}(k)$ and $\operatorname{Im}(k)$ from Eq. (5), (7)), and (99). These explain well the resonance positions on the right range of the black arrow in Fig. 5 (b).

Under the assumption that $\sin \theta$ values of resonance modes distribute uniformly such that $P(\sin \theta)=$ constant, we can obtain the distribution of $\operatorname{Im}(k)$ of resonance modes as

$$
P(\operatorname{Im}(k)) \propto\left(\frac{d(\operatorname{Im}(k))}{d(\sin \theta)}\right)^{-1} .
$$

Using Eq. (5) and (7), we can calculate the distributions for both TM and TE cases, and the results are the red lines in Fig. 4 (a) and (b) which are in a good agreement with the histograms near the minimum imaginary value.

Now we discuss the behavior of resonance positions at the small opening limit, i.e., $n \rightarrow \infty$. Since the imaginary value of a resonance position $k_{( \pm m, l)}$ means the decay rate as shown in Eq. (7), we can safely take the limiting resonance position as a real value $k_{L}$. We first focus on the TM case. When $n$ is very large, the solution of the boundary condition for TM polarization shown in Eq.(4) would be $k=k_{L}+\delta$ where $\delta$ would be a small complex number and be zero at $n \rightarrow \infty$. We can then expand the boundary equation Eq.(4) around $k_{L}$. The Bessel functions can be written as

$$
\begin{aligned}
J_{m}\left(k_{L}+\delta\right) & \simeq J_{m}\left(k_{L}\right)+J_{m}^{\prime}\left(k_{L}\right) \cdot \delta+\cdots, \\
& =J_{m}\left(k_{L}\right)+\left[-J_{m+1}\left(k_{L}\right)+\frac{m}{k_{L}} J_{m}\left(k_{L}\right)\right] \cdot \delta+\cdots,
\end{aligned}
$$

where $J_{m}^{\prime}\left(k_{L}\right)=\left.\frac{d J_{m}(k)}{d k}\right|_{k=k_{L}}$, and the ratio of Hankel functions becomes

$$
\frac{H_{m-1}\left(k_{L} / n\right)}{H_{m}\left(k_{L} / n\right)} \simeq A n^{-1}+i B n^{-2 m+1}
$$

by the approximation by tangents of Bessel functions [15, 16]. The coefficients $A$ an $B$ is given by

$$
\begin{aligned}
& A=\frac{e k_{L}}{2(m-1)}\left(\frac{m-1}{m}\right)^{m-1 / 2}, \\
& B=\frac{1}{2}\left(\frac{e k_{L}}{2}\right)^{2 m-1}\left(\frac{1}{m(m-1)}\right)^{m-1 / 2} .
\end{aligned}
$$

Then, the expanded equation of the boundary condition has small quantities, $1 / n$ and $\delta$, and in the lowest order $(1 / n=0$ and $\delta=0)$, the equation becomes

$$
J_{m-1}\left(k_{L}\right)=0 .
$$


This is nothing but the Dirichlet boundary condition (see Eq.(44)), which means that at the small opening limit the resonance positions with order $m$ of TM case approach the eigenvalues with order $m-1$ of Dirichlet problem, i.e.,

$$
\lim _{n \rightarrow \infty} k_{T M( \pm m, l)}=k_{D( \pm(m-1), l)}
$$

From the equation for the next order, we know how the resonance positions converge to the limiting value $k_{c}$. The result is

$$
\delta_{T M}=-A n^{-2}-i B n^{-2 m}
$$

Note that the imaginary part has an exponent depending on the angular quantum number $m$, while the exponent of the real part does not depend on the mode indices.

For TE polarization, we can obtain similar results. In the lowest order, the TE boundary condition becomes

$$
J_{m}\left(k_{L}\right)=0
$$

This implies

$$
\lim _{n \rightarrow \infty} k_{T E( \pm m, l)}=k_{D( \pm m, l)} .
$$

Note that at the small opening limit the resonance positions with order $m$ of TE case approach the eigenvalues with the same order $m$ of Dirichlet problem. From the equation for the next order, we get

$$
\delta_{T E}=-\frac{k_{L}}{m} n^{-2}-i\left(\frac{k_{L}}{m}\right)^{2} B n^{-2(m+1)} .
$$

From the comparison between $\operatorname{Im}\left(\delta_{T M}\right)$ and $\operatorname{Im}\left(\delta_{T E}\right)$, it is clear that the TE boundary condition is more effective than the TM boundary condition in confining waves inside the circular cavity when the mode incident angle are greater than $\theta_{c}$.

In order to numerically confirm the above results, we trace two resonances of mode indices $(m, l)=(8,1)$ and $(8,40)$ with increasing $n$ for both TM and TE cases. As expected from the analytical results, we obtain

$$
\begin{aligned}
& \lim _{n \rightarrow \infty} k_{T M(8,1)}=k_{D(7,1)} \simeq 11.0864, \\
& \lim _{n \rightarrow \infty} k_{T M(8,40)}=k_{D(7,40)} \simeq 135.6942, \\
& \lim _{n \rightarrow \infty} k_{T E(8,1)}=k_{D(8,1)} \simeq 12.2251, \\
& \lim _{n \rightarrow \infty} k_{T E(8,40)}=k_{D(8,40)} \simeq 137.2123 .
\end{aligned}
$$


In Fig. 6 and Fig. 7, we show the $n$-dependence of $\delta_{T M}$ and $\delta_{T E}$. The results of Eq.(17) and (20), denoted by solid lines, explains well the small opening limit behavior of resonance positions. It is noted that there is a transition point at $n=n_{c} \simeq 17$ in the traces of both $k_{T M(8,40)}$ and $k_{T E(8,40)}$ where the incident angle of the resonance modes(see Eq.(99)) becomes the critical angle $\theta_{c}$ for total internal reflection. Therefore, the transition point indicates the change of leakage mechanism, from refractive to tunneling leakage of waves. For the refractive leakage range $\left(n<n_{c}\right)$, the trace of TM resonance modes shows $1 / n$ dependence [16].

In summary, we have studied the distribution of resonance positions $k_{(m, l)}$ in the circular dielectric cavity, and the behavior of $k_{(m, l)}$ at the small opening limit for both TM and TE polarizations. The distributions of $\operatorname{Im}\left(k_{T M(m, l)}\right)$ and $\operatorname{Im}\left(k_{T E(m, l)}\right)$ are consistent with corresponding ray dynamical SPTD behaviors, and the small opening limits $(n \rightarrow \infty)$ of $k_{T M(m, l)}$ and $k_{T E(m, l)}$ approach different eigenvalues of Dirichlet problem, $k_{D(m-1, l)}$ and $k_{D(m, l)}$, respectively. The result implies that the small opening limit of some open system does not directly match to the corresponding closed system as shown in the TM case.

\section{Acknowledgments}

This work was supported by the Creative Research Initiatives (Center for Quantum Chaos Application) MOST/KOSEF. J.-W. R. and S.-Y. L. were supported by the Brain Korea 21 Project in 2006, and C.-M. K. is partially supported by Sogang Research Grant of 20071114.

[1] R. Blümel, I. H. Davidson, W. P. Reinhardt, H. Lin, and M. Sharnoff, Phys. Rev. A 45 (1992) 2641.

[2] P. A. Chinnery and V. F. Humphrey, Phys. Rev. E 53 (1996) 272.

[3] P. A. Chinnery, V. F. Humphrey, and C. Beckett, J. Acoust. Soc. Am. 101 (1997) 250.

[4] H. -J. Stöckmann and J. Stein, Phys. Rev. Lett. 64 (1990) 2215.

[5] F. Haake, G. Lenz, P. Seba, J. Stein, H. -J. Stöckmann, and K. Zyczkowski, Phys. Rev. A 44 (1991) R6161.

[6] H.-J. Stöckmann, Quantum Chaos; An Introduction (Cambridge University Press, UK, 1999) references therein. 
[7] Optical Processes in Microcavities, edited by R. K. Chang and A. J. Campillo (World Scientific, Singapore, 1996).

[8] C. Gmachl, F. Capasso, E. E. Narimanov, J. U. Nöckel, A. D. Stone, J. Faist, D. L. Sivco, and A. Y. Cho, Science 280 (1998) 1556.

[9] T. Harayama, P. Davis, and K. S. Ikeda, Phys. Rev. Lett. 90 (2003) 063901.

[10] T. Tanaka, M. Hentschel, T. Fukushima, and T. Harayama, Phys. Rev. Lett. 98 (2007) 033902.

[11] J.-M. le Floch, J. D. Anstie, M. E. Tobar, J. G. Hartnett, P.-Y. Bourgeois, and D. Cros, Phys. Lett. A 359 (2006) 1.

[12] F. Borgonovi, I. Guarneri, and D. L. Shepelyansky, Phys. Rev. A 43 (1991) 4517.

[13] J.-W. Ryu, S.-Y. Lee, C.-M. Kim, and Y.-J. Park, Phys. Rev. E 73 (2006) 036207.

[14] J. D. Jackson, Classical Electrodynamics 2nd Edition (John Wiley \& Sons, New York, 1975).

[15] I. S. Gradshteyn, and I. M. Ryzbik, Table of Integrals, Series, and Products, 6th Edition (Academic Press, San Diego, 2000).

[16] J. U. Nöckel, Ph.D. thesis, Yale University, 1997.

[17] M. Hentschel, Ph.D. thesis, Max Planck Institute for the Physics of Complex Systems, 2002.

[18] M. Hentschel and J. U. Nöckel, physics/0203064 (2002).

[19] J. Hawkes and I. Latimer, Lasers; Theory and Practice (Prentice Hall, Englewood Cliffs, NJ, 1995). 


\section{Figure Captions}

FIG. 1: The intensity patterns of a mode $(m, l)=(8,1)$ for various boundary conditions; (a) Dirichlet, (b) Neumann, (c) TM, and (d) TE boundary conditions.

FIG. 2: The intensity pattern of the additional resonance mode $( \pm m, l)=( \pm 8, \times)$ in the TE case. This corresponds to a very leaky mode showing wave escaping through the Brewster angle $\theta_{B}$.

FIG. 3: The level spacing distributions. (a) TM case. (b) TE case. These show good agreements with Poisson distribution (the solid line).

FIG. 4: The imaginary value distributions of resonance positions when $n=2$; (a) TM case and (b) TE case. Red lines are the results of a semiclassical analysis, Eq.(10).

FIG. 5: (a) The minimum imaginary values of the distributions for $n=2,4,6,8,10$ in TM case are denoted by solid dots. The solid line represents $-\gamma_{M}(n, \theta=0) / 2$ and $\gamma_{M}(n, \theta=0)$ (see Eq. (8) ) is the decay rate of bouncing ball trajectory. (b) The resonance positions with a fixed angular quantum number, $m=40$, and $n=2$ for both TM (rectangles) and TE (diamonds) cases. The left and right arrows represent $\operatorname{Re}(k)$ values where the incident angle of resonance modes (see Eq. (9) ) becomes to the critical angle $\theta_{c}$ and the Brewster angle $\theta_{B}$, respectively. The resonance positions with incident angle greater than $\theta_{c}$ are well explained by results of the semiclassical analysis described by green (TM) and blue (TE) solid lines. Red cross represents an additional resonance mode which is originated from the existence of the Brewster angle.

FIG. 6: The approaching behaviors of real part of the resonance positions corresponding to mode indices $(m, l)=(8,1)$ and $(8,40)$ with increasing $n$ (the small opening limit). The solid lines are the real parts (first term) of Eq.(17) and (20) showing $n^{-2}$ behavior. FIG. 7: The approaching behaviors of imaginary part of the resonance positions corresponding to mode indices $(m, l)=(8,1)$ and $(8,40)$ with increasing $n$ (the small opening limit). The solid lines are the imaginary part (second term) of Eq.(17) and (20) showing $n^{-16}$ (TM) and $n^{-18}(\mathrm{TE})$. 


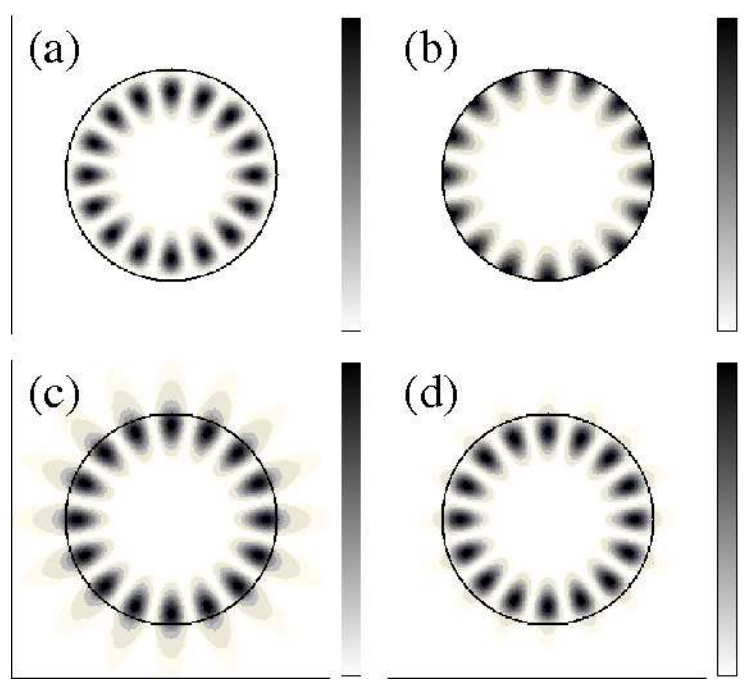

FIG. 1:

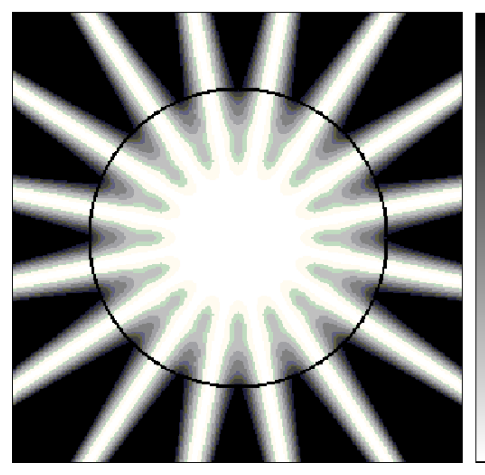

FIG. 2: 

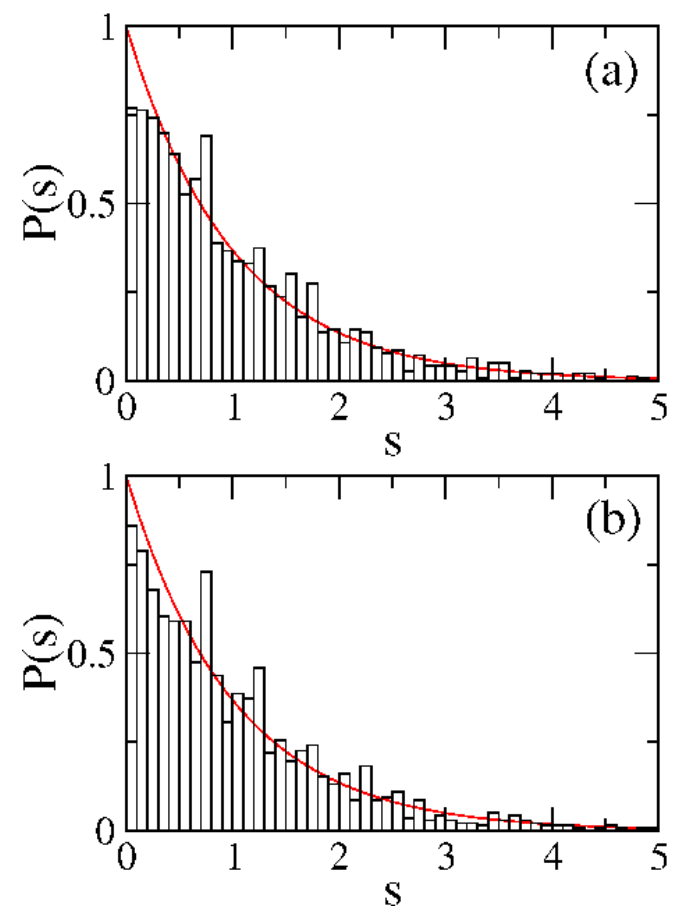

FIG. 3:
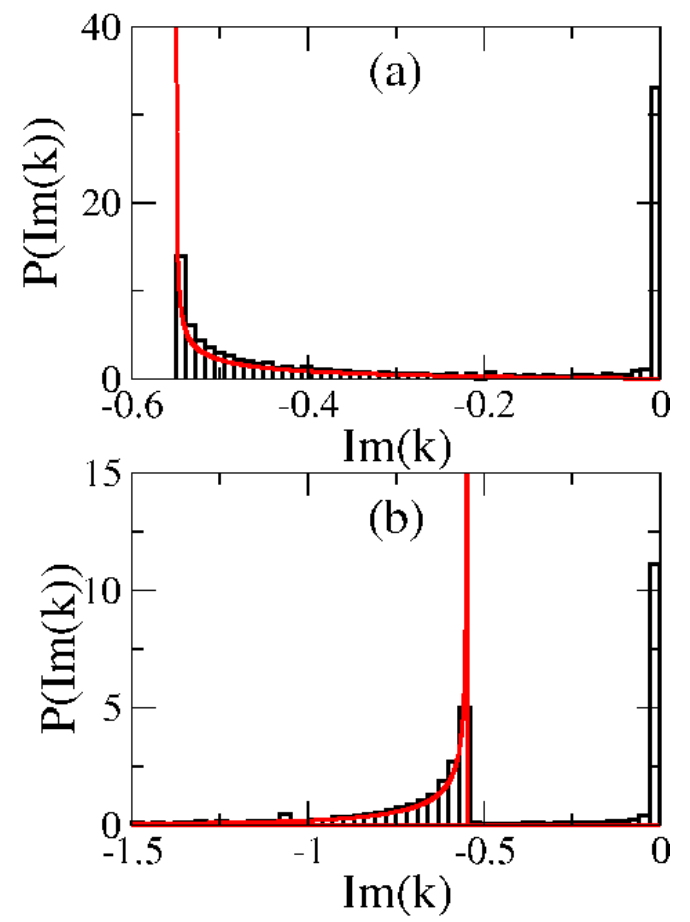

FIG. 4: 

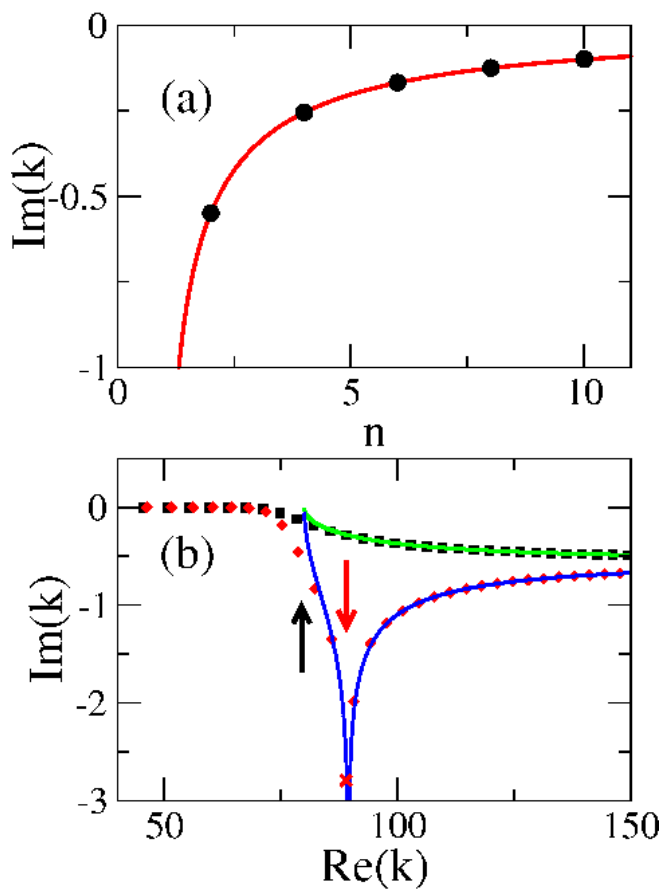

FIG. 5:

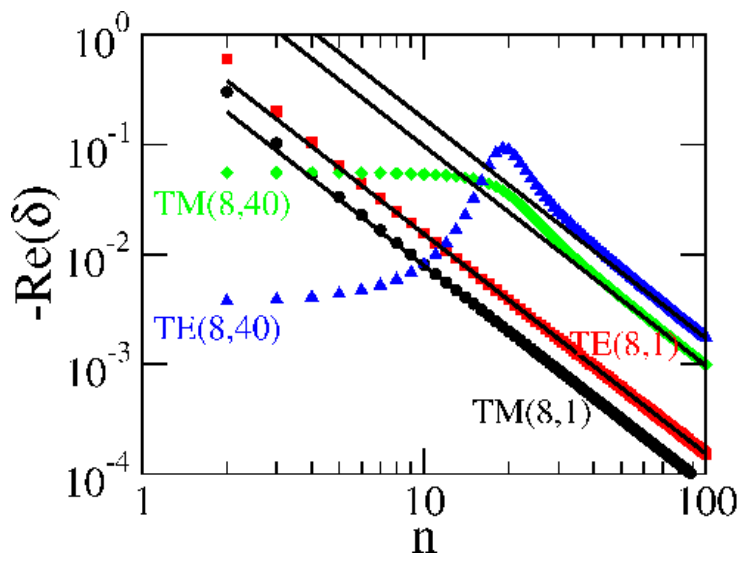

FIG. 6: 


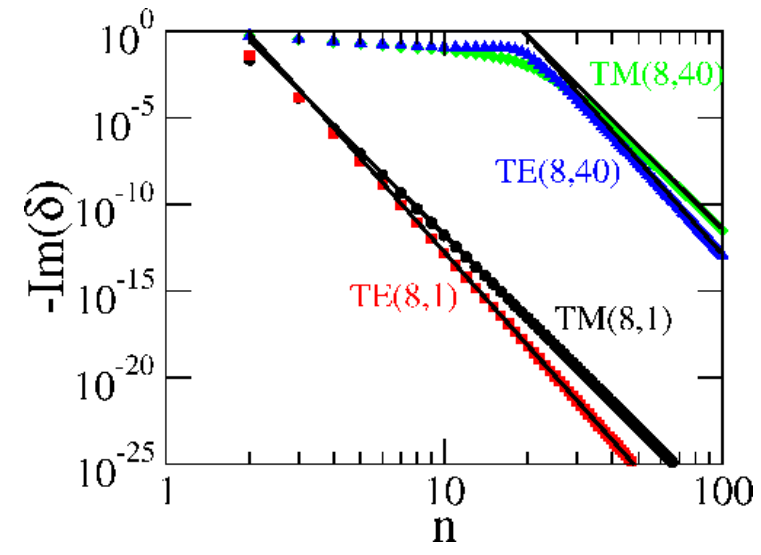

FIG. 7: 